\title{
Correction to: Does executive function capacity moderate the outcome of executive function training in children with ADHD?
}

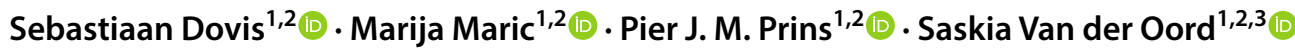

Published online: 21 June 2019

(c) Springer-Verlag GmbH Austria, part of Springer Nature 2019

\section{Correction to:}

\section{ADHD Attention Deficit and Hyperactivity Disorders} https://doi.org/10.1007/s12402-019-00308-5

The original version of this article unfortunately contained a mistake. The captions of Figures 1 and 2 were swapped.

Figures 1 and 2 with corrected captions are given in the following page.

The original article has been corrected.

The original article can be found online at https://doi.org/10.1007/ s12402-019-00308-5.

Saskia Van der Oord

saskia.vanderoord@kuleuven.be

1 Developmental Psychology, University of Amsterdam,

Nieuwe Achtergracht 129B, 1001 NK Amsterdam,

The Netherlands

2 Cognitive Science Center Amsterdam, University of Amsterdam, Nieuwe Achtergracht 129B, 1001 NK Amsterdam, The Netherlands

3 Clinical Psychology, KU Leuven, Tiensestraat 102, bus 3720, 3000 Leuven, Belgium 
Fig. 1 Conceptual moderation model. Note: BRIEF Behavior Rating Inventory of Executive Function questionnaire, CBTT Corsi block-tapping task, DBDRS Disruptive Behavior Disorder Rating Scale, Far transfer measures that assess constructs or domains different from the trained tasks, Near transfer measures similar to the trained tasks in terms of format and processing requirements, RCI Reliable Change Index (pre- to post- and pre- to follow-up RCIs were used), STM short-term memory, TMT trail-making task, $W M$ working memory

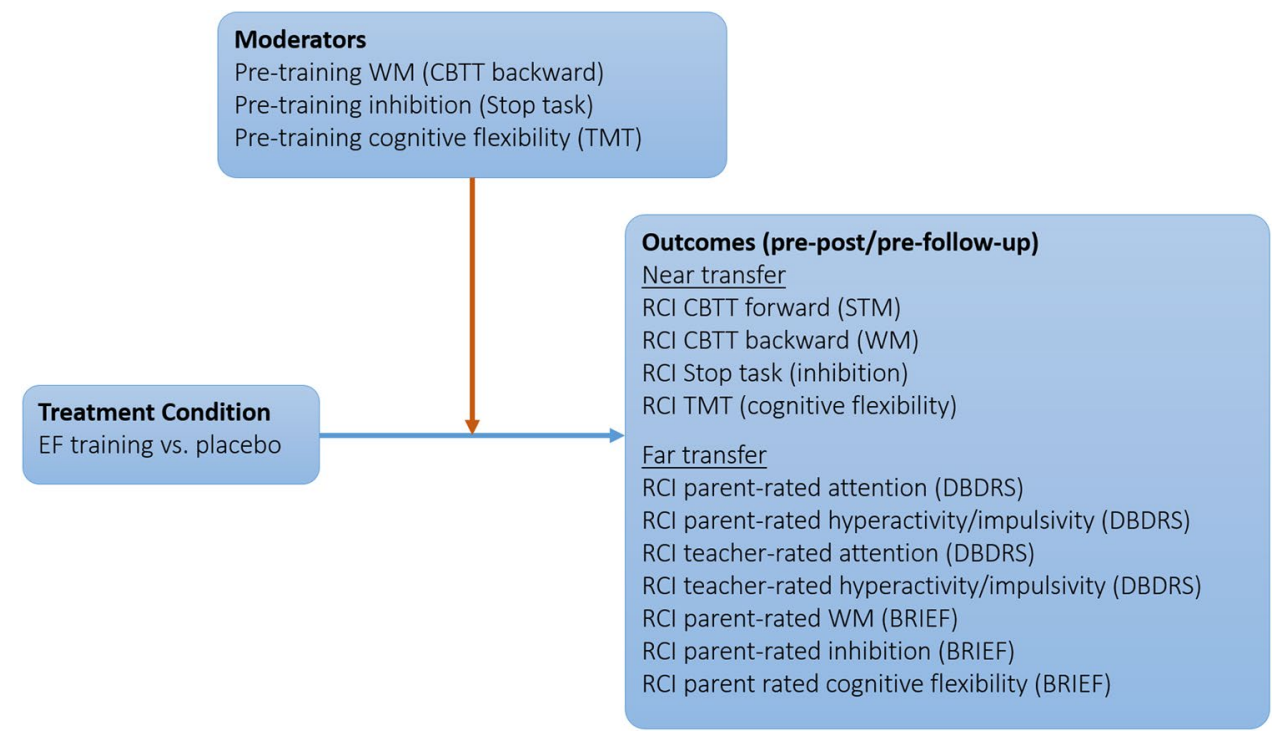

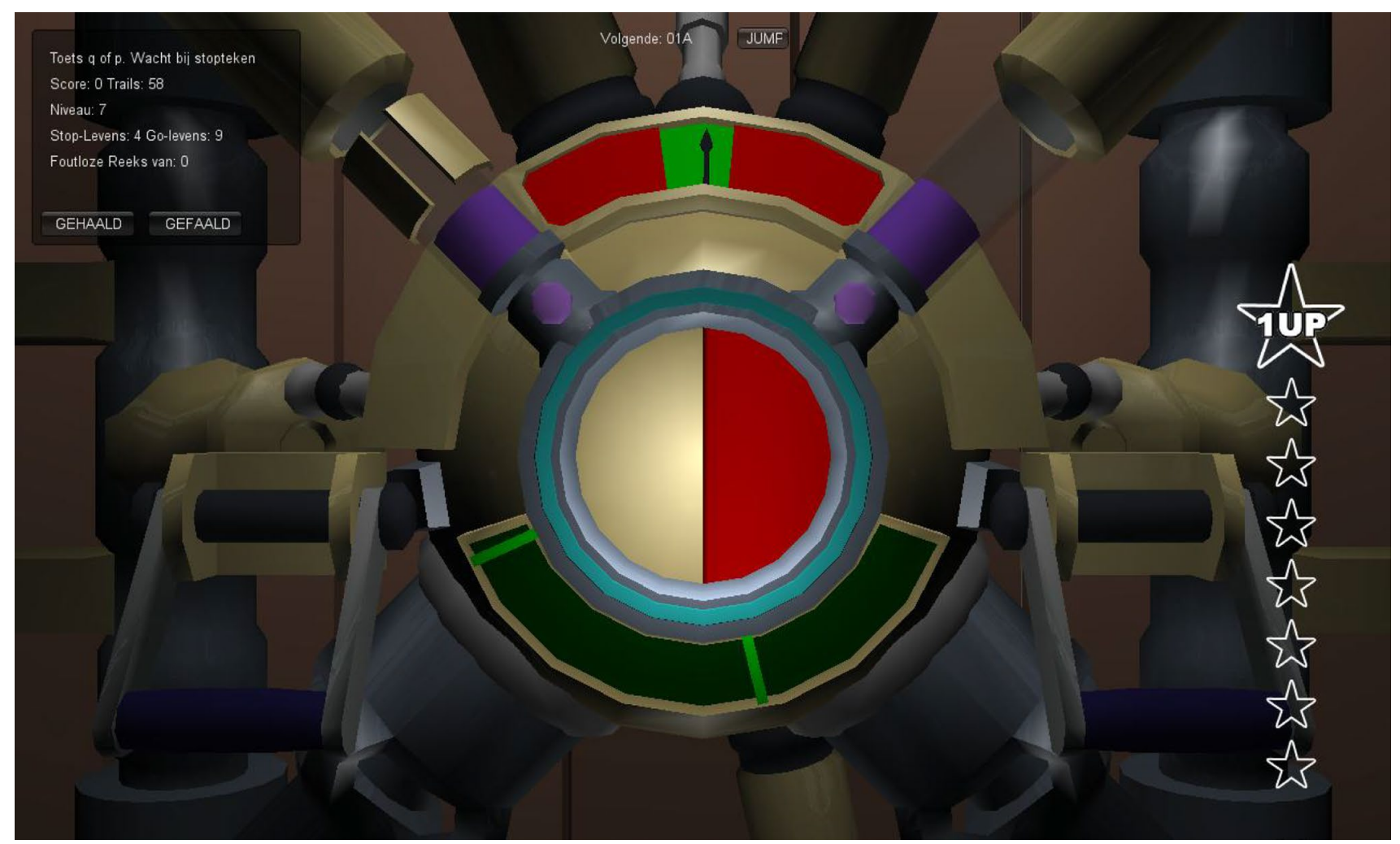

Fig. 2 The inhibition training task with the green-colored time frame (response window) in the upper middle of the screen 